\title{
Myzetome in Ostafrika - Langzeitverläufe und späte Komplikationen
}

\author{
Mycetomas in East Africa - Long-Term Clinical Course and Late Complications
}

Autoren

Institut
V. Orfanos, J. Masenga, M. Ketema, C. E. Orfanos

Regional Dermatology Training Centre (RDTC), Kilimanjaro Christian Medical College, Tumaini Universität, Moshi, Tansania

\section{Bibliografie}

DOI $10.1055 / \mathrm{s}-2006-944931$

Akt Dermatol 2006; 32;

523-526 @ Georg Thieme

Verlag KG Stuttgart · New York ISSN 0340-2541

Korrespondenzadresse John Masenga, M.D., Principal Regional Dermatology Training Centre, Kilimanjaro Christian Medical College (KCMC)

P.O. Box 8332

Moshi · Tanzania

johnmasenga@yahoo.com

\section{Zusammenfassung \\ $\nabla$}

Es werden zwei Kranke mit Eumyzetom in Ostafrika beschrieben, die den langjährigen Verlauf und die damit verbundenen Komplikationen dieser Infektion mit Madurella mycetomatis und anderen Bodensaprophyten demonstrieren sollen. Kennzeichnend für beide war der harmlose Beginn mit indolenten Papeln an den Füßen, sowie der lange Verlauf bis zum Auftreten der ersten Beschwerden. In der Regel veranlassen erst letztere die Kranken den Arzt aufzusuchen, meist in einem fortgeschrittenen Stadium mit tumorösen Granulomen, die infiltrativ in die Tiefe wachsen. Die Behandlung wird dadurch für den Arzt

\section{Einleitung}

$\nabla$

Myzetome entstehen nach Infektionen mit Schimmelpilzen (Eumyzetom) oder Bakterien bzw. anderen Erregern (Aktinomyzetom), wobei Mischformen nicht selten sind. Eumyzetome kommen häufiger in subtropischen und tropischen Klimazonen in Afrika und Asien vor (Mittlerer Osten, Subsahara, Indien), während Aktinomyzetome in nordafrikanischen Ländern (Tunesien, Marokko, Senegal), öfters aber in Mittelund Südamerika auftreten. In beiden Fällen sind vorwiegend Farmer und Landarbeiter betroffen, deren ungeschützte Haut mit der Erde in Kontakt kommt, z. B. beim barfuß Laufen. Die häufigsten Erreger sind beim Eumyzetom Madurella mycetomatis und Madurella grisea, beim Aktinomyzetom hingegen Actinomadura madurae, Madura streptomyces und verschiedene Nocardia-Spezies. Mischinfektionen mit weiteren Schimmelpilzarten und Bodensaprophyten sind häufig.

Nach Bagatellverletzungen der Haut durch Dornen, Splitter etc. können verschiedene Bodensaprophyten in die Subkutis inokuliert werden. Nach langer Inkubationszeit, oft erst nach vielen schwierig und bleibt oft unbefriedigend. Ist noch keine Knochendestruktion nachweisbar, so sind Breitspektrum-Antimykotika aus der Gruppe der Azole bzw. Terbinafin das Mittel der Wahl, möglichst mit operativer Entfernung der tumorösen Granulome. Sind die darunter liegenden Knochen bereits angegriffen oder gar das Knochengerüst zerstört, kommt nur noch eine Amputation des nicht mehr funktionstüchtigen Körperteils infrage. Die Infektion ist in Ostafrika relativ häufig, insbesondere bei Farmern, Land- und Plantagenarbeitern, die oft barfuß in den Feldern ihre Arbeit verrichten. Eine entsprechende Aufklärung fehlt sowohl bei den Betroffenen als auch bei ihren Arbeitgebern.

Monaten, entstehen an der betroffenen Stelle am Fuß, seltener an anderen Körperstellen, schmerzlose, indurierte subkutane Papeln, danach Knoten, wobei im weiteren Verlauf es zu einer lokalisierten, chronisch-progressiven, entzündlichgranulomatösen Reaktion der Haut und im subkutanen Fettgewebe kommt, die weiter symptomlos bleibt. Im weiteren Verlauf wird später auch die darunter liegende Muskulatur bzw. der Knochen erfasst [2], so dass Beschwerden auftreten. Häufige Folgen eines länger bestehenden, nicht behandelten Myzetoms sind Periostitis, Osteomyelitis und Knochendestruktion, die therapeutisch schwer angehbar sind [5]. Der betroffene Fuß zeigt eine teigige, später indurierte und phlegmonöse Schwellung, mit nässenden Fisteln und - je nach Erreger - schwarze, weißliche oder auch gelbe Granulae, die oft bereits makroskopisch an der Oberfläche der Läsionen erkennbar sind, und sich bei der mikroskopischen Untersuchung als größere Knäuel von Pilzen und Saprophyten bzw. Aktinomyzeten erweisen. Beim weiteren Verlauf der Erkrankung, für Therapie und Prognose ist eine Differenzierung des Erregers sinnvoll. Während das Aktinomyzetom auf einige 
Antibiotika in der Regel gut anspricht, erweist sich die Behandlung des Eumyzetoms als schwierig und meist unbefriedigend. Während unserer mehrmonatigen ärztlichen Tätigkeit in Ostafrika, im Kilimanjaro Christian Medical College im Norden Tansanias, haben wir mehrere Myzetome sehen und behandeln können, die vom klinischen Bild und ihrem Verlauf her eindrucksvoll gewesen sind. Hier wird exemplarisch über zwei Fälle mit langjährigem, komplizierten Verlauf berichtet.

\section{Klinische Kasuistik \\ $\nabla$}

\section{Fall 1}

Die Patientin: A. E., geb. 1970, aus der ländlichen Gegend von Mbuguni in der Nähe von Arusha (File-Nr. 57-34-71), stellte sich erstmals am 5.1.1999 in unserer Ambulanz vor.

Zur Anamnese gab sie an, dass sie seit 1985 rezidivierende Schwellungen des rechten Fußes im Außenknöchelbereich hatte, die sie nicht weiter beachtete. Mehrere Jahre später hätten die Schwellungen zugenommen und der Fuß habe genässt. Erst nachdem sich der Befund erheblich verschlechtert hatte, habe sie sich entschlossen, die Klinik aufzusuchen. Zum damaligen Zeitpunkt wurden im Bereich des rechten lateralen Malleolus eine weich-teigige Schwellung mit einer Flüssigkeit absondernden Fistel, einzelnen kleinen Ulzera und makroskopisch sichtbaren schwarz-braunen Granulae festgestellt. Eine Röntgenaufnahme des rechten Fußes zeigte eine Weichteilschwellung ohne Knochenbeteiligung. Der Patientin wurden Cloxacillin $(2 \times 500 \mathrm{mg} /$ die $)$, Paracetamol und Kaliumpermanganat-Fußbäder verordnet und ihr empfohlen, zwecks weiterer Maßnahmen sich stationär einweisen zu lassen. Erst einige Monate später wurde die Pat. mit dem Verdacht auf ein Eumyzetom stationär aufgenommen.

Diagnostik: Im Abstrichmaterial wuchsen Streptokokken und Staphylokokken, Aktinomyceten wurden nicht nachgewiesen. Im Aspirat aus dem Fistelgang wurden unter anderem Riesenzellen gefunden, die septierte Organismen enthielten. Somit wurde die Diagnose tiefe Mykose, am ehesten Eumyzetom, gestellt. Die Histologie eines Biopsats aus der rechten Ferse zeigte in multinukleären Histiozyten bzw. in Riesenzellen eingeschlossene, verzweigte, gelb-orange Einschlüsse, am ehesten Pilzelemente, die auch im Fistelgang in der Dermis und in der hyperkeratotischen Epidermis nachweisbar waren. Die Diagnose Myzetom (Eumycetom) mit Fistelbildung wurde bestätigt. Ultraschall-Untersuchungen vom rechten Fuß ließen 4 subkutane, knotige Infiltrate ohne tiefe Fistelbildung erkennen, einige davon schienen fibrosiert und abgeheilt. Ein tiefer Abszess war nicht nachweisbar. Die üblichen Laborwerte waren weitgehend unauffällig. Verlauf und Therapie: Die Patientin bekam daraufhin $200 \mathrm{mg}$ Itraconazol/d, das sie gut vertrug. Sie wurde aus der stationären Behandlung entlassen und wurde bis September 2005 nicht mehr gesehen. Erst am 29. 9. 2005 erschien sie erneut in unserer Poliklinik. Der gesamte rechte Fuß war zu diesem Zeitpunkt stark angeschwollen, ein großer, exophytisch wachsender Tumor führte zu einer erheblichen Beeinträchtigung beim Laufen. Es wurde erneut die Diagnose Madurella-Myzetom gestellt. Da die Patientin zu diesem Zeitpunkt im 9. Monat schwanger war und keine Medikamente einnehmen wollte, wurde auf eine gezielte medikamentöse Behandlung verzichtet und eine stationäre Aufnahme nach Beendigung der Schwangerschaft vereinbart.

Am 1. 2. 2006 wurde die Pat. von uns gesehen und erneut stationär aufgenommen. Der gesamte rechte Fuß war zu diesem Zeit-

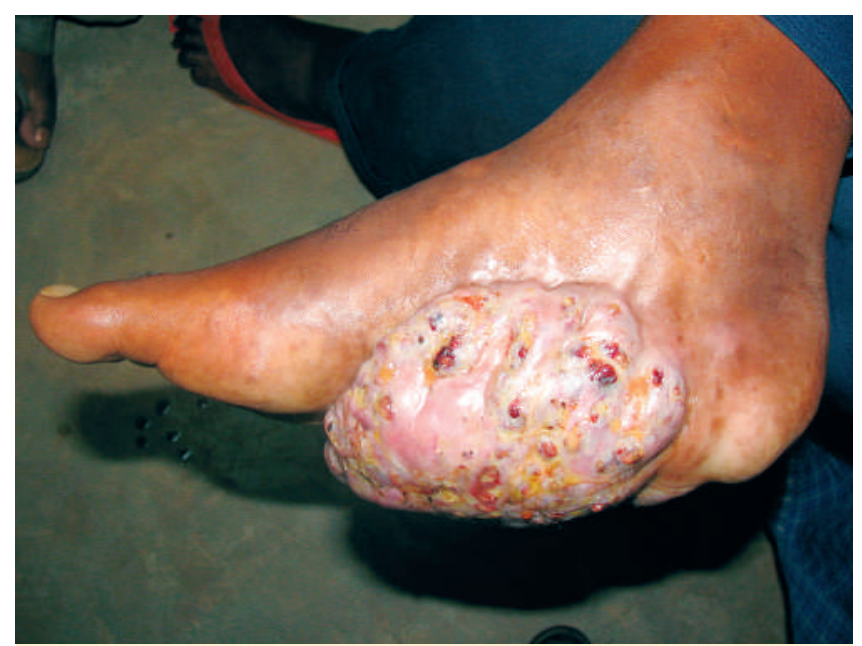

Abb. 1 Fall 1: 36-jährige Patientin mit Riesen-Eumyzetom an der Innenseite des rechten Fußes, seit mehreren Jahren wachsend.

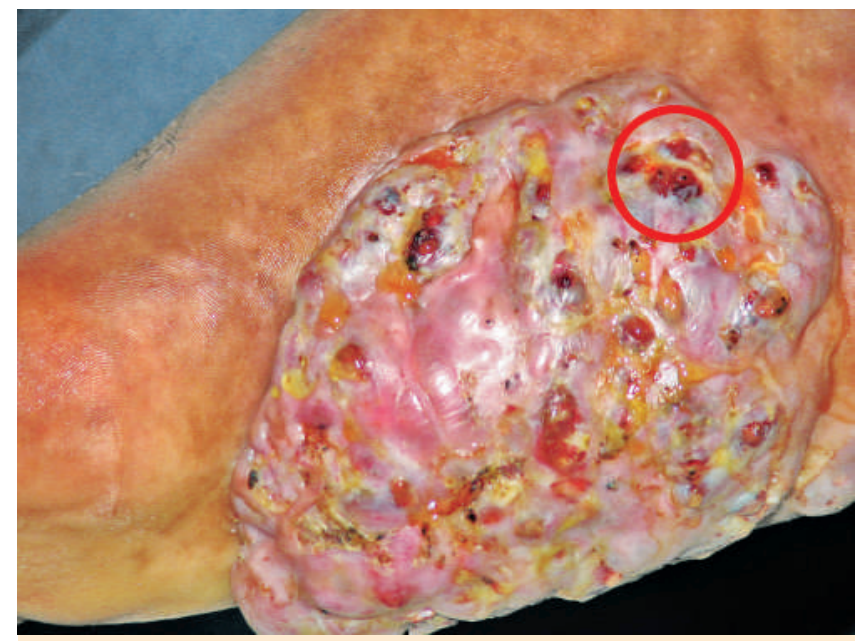

Abb. 2 Fall 1: An der Oberfläche der tumorösen Läsion sind die schwarzen granulären Einschlüsse gut erkennbar (roter Kreis), die für die Diagnose eines Eumyzetoms charakteristisch sind.

punkt stark angeschwollen, latero-plantar fanden wir einen kindskopfgroßen, zerklüfteten, blumenkohlartigen Tumor ( $\bullet$ Abb. 1), mit gut sichtbaren Fistelgängen und schwarzen Granulae an seiner Oberfläche ( $\bullet$ Abb.2). Da die Patientin darauf bestand, ihren 4 Monate alten Säugling weiter zu stillen, haben wir zunächst auf orale Pharmaka verzichtet und die operative Entfernung des riesigen Myzetoms empfohlen. Die röntgenologische Untersuchung des Knochens ließ keine Läsionen erkennen, die Laborwerte waren unauffällig. Am 28.2.2006 wurde die Pat. operiert, wobei die Wunde nicht primär verschlossen werden konnte. Da wir beim ersten postoperativen Verbandswechsel noch schwarze Granulae am Wundgrund fanden, sind wir davon ausgegangen, dass eine In-toto-Ausräumung der tumorösen Granulome nicht gelungen war. Der Patientin wurde daraufhin empfohlen, mit dem Stillen aufzuhören, um eine höher dosierte orale Therapie mit Itraconazol durchführen zu können. Sie beschloss, erst mit ihrem Ehemann darüber zu sprechen, und verließ unser Krankenhaus. 
Fall 2

Der Patient M. P., geb. 1977, stammt aus einer ländlichen Gegend von Igunga bei Tabora (File-Nr. 72-80-21). Der 28-jährige junge Mann wurde am 28.12. 2005 wegen des Verdachts auf ein Myzetom stationär aufgenommen.

Zur Anamnese gab er an, dass er vor 6 Jahren am linken Fuß einen persistierenden Juckreiz bemerkte, und seit 5 Jahren eine zunehmende, schmerzlose Schwellung mit fistulösen Gängen und Absonderungen von Flüssigkeit. Aus diesem Grunde suchte er während der Jahre 2001 und 2002 einen „traditionellen Heiler“ in seinem Heimatort auf, der ihn mit Kräuterextrakten behandelte. Eine Besserung trat aber auch nach mehreren Monaten nicht auf, so dass er sich im November 2005 im Regional-Krankenhaus von Tabora vorstellte, wo er über 1 Monat orale Gaben von Ketoconazol erhielt. Da auch darunter keine Besserung eintrat, wurde der Patient von dort aus in die Abteilung für Dermatologie des KCMC in Moshi verlegt.

Befund und Diagnostik: Bei der Vorstellung des Patienten fanden wir eine starke Schwellung des linken Fußrückens mit Fisteln, die Flüssigkeit absondernde und makroskopisch sichtbare schwarze Granulae an der Oberfläche aufwiesen. Das Fußgelenk war deformiert und der linke Fuß in eine extreme Spitzfuß-Stellung geraten ( Abb.3). Bei der röntgenologischen Untersuchung zeigten alle tarsalen und metatarsalen Knochen und die distalen Tibia- und Fibulaanteile ein stark verdichtetes Knochengerüst, mit deutlichen und ausgedehnten osteolytischen Herden. Die Tarso-metatarsal-Gelenke waren im Rahmen eines tief greifenden Entzündungsprozesses völlig zerstört. Bei den Laboruntersuchungen waren sämtliche Werte bis auf ein erniedrigtes Hämoglobin weitgehend unauffällig.

Therapie: Wegen der ausgedehnten Knochendestruktionen und der Fehlstellung des betroffenen Fußes erschien eine konservative medikamentöse Behandlung allen behandelnden Ärzten nicht mehr sinnvoll. Dem Patienten musste die Amputation des linken Fußes empfohlen werden, wozu er seine Zustimmung gab. Am 7. 2. 2006 wurde der linke Unterschenkel unterhalb des Knies, weit im Gesunden amputiert. Postoperativ erhielt er Cloxacillin $1 \mathrm{~g} / \mathrm{d}$ über mehrere Wochen, um eine evtl. verbleibende Osteomyelitis vorbeugend zu behandeln. Die Operationswunde heilte primär $a b$, und der junge Mann konnte Anfang März 2006 die Klinik verlassen, mit dem Hinweis, sich nach zwei Monaten erneut vorzustellen, um sich eine entsprechende Prothese anpassen zu lassen.

\section{Diskussion}

$\nabla$

Eumyzetome kommen in Ostafrika offenbar relativ häufig vor. Innerhalb von 3 Monaten wurde von uns diese in Europa sehr seltene Infektion bei 5 Patienten diagnostiziert. Alle Patienten waren jung, kamen aus ländlichen Gebieten, wo sie, meist barfuß, auf den Feldern arbeiteten. In allen Fällen, die wir gesehen haben, war das Eumyzetom am Fuß lokalisiert und bereits in sehr fortgeschrittenem, tumorösem Stadium. Allen Kranken war gemeinsam, dass zwischen den ersten Hautveränderungen in Form von schmerzlosen Knoten und der ersten Konsultation in tumorösem Stadium eine große Zeitspanne, meist Jahre, lagen.

Das Eumyzetom ist eine tückische, aber vermeidbare Erkrankung. Die Infektion entwickelt sich langsam, über einen langen Zeitraum, ohne Beschwerden, so dass sie zunächst nicht ernst genommen wird. In Ostafrika ist außerdem der Zugang zu einem

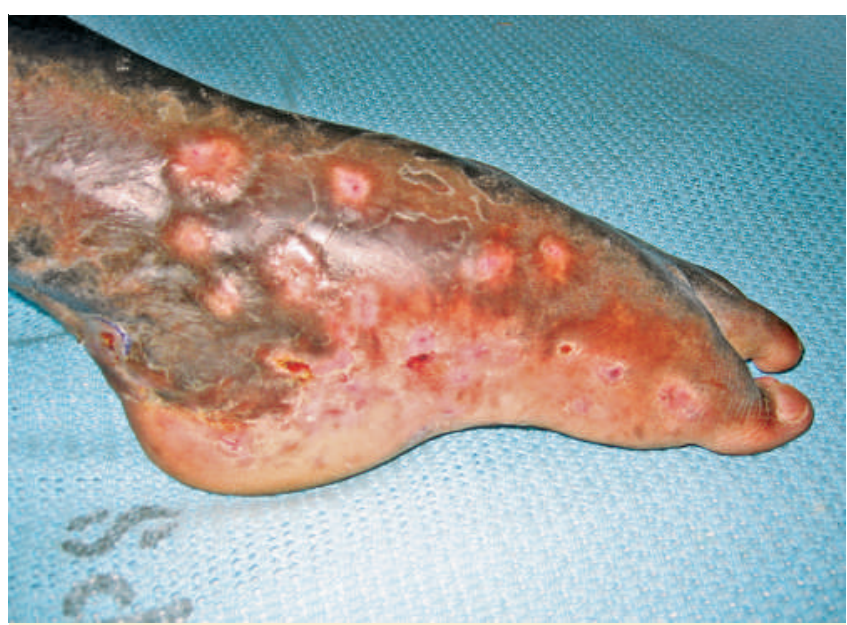

Abb. 3 Fall 2: Linker Fuß eines 28-jährigen Mannes, der bereits seit vielen Jahren an einem Eumyzetom leidet, mit Ketokonazol teilbehandelt. Röntgenologisch zeigte sich eine fortgeschrittene Destruktion des gesamten Sprunggelenkes einschließlich der Malleolargegend, so dass eine Amputation unvermeidlich war.

Arzt, insbesondere zu einem Dermatologen, schwierig. Hier spielen geringe Arztdichte und weite Entfernungen eine große Rolle. Kaum ein Patient wird wegen schmerzloser Knoten am Fuß, die ihn nicht weiter stören, die Mühen und Kosten eines Arztbesuchs auf sich nehmen. Die Patienten kommen erst, wenn die Krankheit weit fortgeschritten ist. Nach langsamem Wachstum in die Tiefe kommt es zu Knochendestruktionen, wie in unserem zweiten Fall. Sind die darunter liegenden Muskelgruppen und das Knochengerüst durch die Tumormassen zerstört und der Fuß deformiert, bleibt als letzter Ausweg oft nur eine Amputation.

Eine gründliche Aufklärung der ländlichen Bevölkerung über die Existenz und das Wesen dieser Krankheit wäre wünschenswert, verbunden mit dem guten Rat, bei Feldarbeiten solides Schuhwerk zu tragen. Während unseres Aufenthaltes in Tansania haben wir Plantagenbesitzer (Kaffee, Blumen) darauf aufmerksam gemacht, dass sie für solche Vorsichtsmaßnahmen bei ihren Landarbeitern Sorge tragen müssten.

Die medikamentöse Behandlung des Eumyzetoms ist schwierig und weniger befriedigend als die des Aktinomyzetoms. Wird der Patient jedoch rechtzeitig einer gezielten antimykotischen Behandlung mit Azolen (in der Regel Itrakonazol $400 \mathrm{mg} / \mathrm{d}$ über 8 Wochen, Fortführung bzw. Reduzierung der Dosis je nach klinischer Ansprechbarkeit und Toxizität), ggf. in Kombination mit der operativen Entfernung der Primärläsion, zugeführt, bestehen gute Aussichten auf einen Heilungserfolg $[2,4,5]$. Auch Terbinafin $(2 \times 500 \mathrm{mg} / \mathrm{d}$ über 24 Wochen oder länger) hat sich als wirksam erwiesen [6,7]. Bei Mischinfektionen kommen Kombinationen mit anderen Medikamenten (Amphotericin B, Trimethoprim-Sulfamethoxazol, Rifampicin, Amicacin, u.a.) infrage. Für refraktäre Fälle wurde neuerdings Posakonazol empfohlen [8]. Unsere erste Patientin kam erst 14 Jahre nach der Erstinfektion zur ärztlichen Konsultation bzw. zur Behandlung. Sie erhielt während des stationären Aufenthalts über 6 Wochen Itraconazol in mittlerer Dosierung ( $200 \mathrm{mg} /$ p. o.), doch ob und wie lange diese Medikation nach der Entlassung fortgeführt wurde, war nicht zu eruieren. Innerhalb der folgenden 6 Jahre entwickelte sich ein großes tumoröses Myzetom am rechten Fuß, wahrscheinlich, weil die Behandlung nicht ordnungsgemäß weitergeführt wor- 
den war. Es kam dazu, dass wir wegen der Schwangerschaft und der langen Stillperiode auf eine orale Therapie mit Azolpräparaten verzichtet haben, auch wenn eine Teratogenität der Azole im Tierexperiment (Ratten, Mäuse) beim Menschen nicht wahrscheinlich ist $[1,9]$.

Ob die operative Entfernung der Granulome, die meist in fortgeschrittenem Stadium erfolgt, zur Heilung führt, bleibt von Fall zu Fall umstritten, je nachdem ob eine chirurgische Totalentfernung der infizierten Gewebsanteile gelingt. Unter anderem wurde berichtet, dass eine möglichst frühzeitige operative Entfernung der ersten auftretenden Läsionen, in Kombination mit oralen Itraconazol- oder Terbinafin-Gaben die wirksamste Behandlung eines Myzetoms darstellt [6].

\section{Danksagung \\ $\nabla$}

Dr. med. Vera Orfanos und Prof. emer. Dr. C. E. Orfanos waren als Guest Physicians and Lecturers im RDTC/KCMC.

\section{Abstract}

\section{Mycetomas in East Africa - Long-Term Clinical Course and Late Complications \\ $\nabla$}

Two cases of eumycetoma seen in East Africa are reported, demonstrating the clinical course and the possible complications of this infection with Madurella mycetomatosis and other earth saprophytes. Most characteristic for both cases was the harmless onset of the disease, with indolent papules and nodules on the feet, and the progressive long-term course with tumorous granulomas growing in the deeper dermis and subcutis and increasing discomfort. Most patients consult a physician in progressed stages showing tumorous lesions infiltrating in deeper tissues. In these late cases management is difficult and often remains unsatisfactory. If no bone destruction has already occurred, long-term administration of broad spectrum azoles or terbinafine in higher dose levels are recommended for treatment. In cases with progressed tumorous infiltrates surgical removal should be also performed. If the underlying bone tissue is affected and destroy, however, amputation seems unavoidable. The infection appears rather common in East Africa, particularly in farmers, agricultural labor workers and planters, who work with unprotected feet in their fields. Appropriate information is obviously lacking both among the workers and their employers.

\section{Literatur}

1 Bar-Oz B, Moretti ME, Bishai R, Mareels G, van Tittelboom T, Verspeelt J, Koren G. Pregnancy outcome after in utero exposure to itraconazole: a prospective cohort study. Am J Obstet Gynecol 2000; 183: 617-620

2 Brownell I, Pomeranz M, Ma L. Eumycetoma. Dermatol Online J 2005; 11: 10

3 Daoud M, Ezzine Sebai N, Badri Tet al. Mycetoma: retrospective study of 13 cases in Tunesia. Acta Dermatovenerol Alp Panonica Adriat 2005; $14: 153-156$

4 Develoux M, Dieng MT, Kane A, Ndiaye B. Management of mycetoma in West-Africa. Bull Soc Pathol Exot 2003; 96: 376- 382

5 Dieng MT, Niang SO, Diop B, Ndiaye B. Actinomycetomas in Senegal: a study of 90 cases. Bull Soc Pathol Exot 2005; 98: 18-20

6 Hay RJ. Therapeutic potential of terbinafine in subcutaneous and systemic mycoses. Br J Dermatol 1999; 56: 36-40

7 Ndiaye B, Dieng MT, Perez A, Stockmeyer M, Bakshi R. Clinical efficacy and safety of oral terbinafine in fungal mycetoma. Int $\mathrm{J}$ Dermatol 2006; 45: $154-157$

8 Negroni R et al. Posaconazole treatment of refractory eumycetoma and chromoblastomycosis. Rev Inst Med Trop Sao Paulo 2005; 47: 339346

9 Van Canteren H, Lampo A, Vandenberghe J et al. Safety aspects of oral antifungal agents. Br J Clin Pract suppl 1990; 71: 47-49

\section{Buchbesprechung}

\section{Netters Dermatologie}

F. H. Netter

Stuttgart: Thieme, 2006. 416 S., 183 Farbtafeln. Geb. 34,96€

ISBN 3-13-141001-9

Der neu erschienene Taschenatlas der Dermatologie bietet anhand einprägsamer Darstellungen eine einzigartige Einführung in die Dermatologie. Mehr als 600 klinische Bilder auf fast 200 Bildtafeln sowie eingestreut klinische Fotos geben einen gekonnten Einblick in die dermatologische Welt. Anatomie, Physiologie und Klinik werden dabei auf einer Bildtafel vereint. Kurze Texte bieten die notwendige Erklärung. Dem Buch merkt man seine Historie an: Generationen von Ärzten wurde durch die Netter-Farbatlanten zur Medizin geholfen, in die teilweise komplizierte medizinische Materie und deren Zusammenhänge einzudringen.
Gerade visuell veranlagte Kollegen werden die bildliche Darstellung zu schätzen wissen, vereinfacht sich doch der Lernprozess erheblich. Die Historiker unter uns erhalten ein sorgsam aufbereitetes Werk, das zeigt, dass auch in früheren Jahren wenngleich mit erheblich mehr Mühe verbunden - ein Unterricht mit didaktisch hochwertig aufbereiteten Büchern stattfand. Ein derartiges Paradepferd liegt hier vor. Lernen ist stets mit Mühe verbunden, warum also und nicht noch einmal sein Wissen auf Basis des Netters Dermatologie auffrischen?

M. Herbst, Heidelberg 